\title{
Erfahrungen bei der Definition nationaler Qualitätsindikatoren für die Schweiz
}

D. Zahnd

Qualitätsmanagement

Schlüsselwörter

Behandlungsqualität im Spital

Routinedaten

Key words

Quality of care

inpatients

routine data

patient minimum data set

\section{Ausgangslage}

In der Schweiz war die Umsetzung der Qualitätssicherung in den Spitälern bisher im Wesentlichen den Tarifpartnern (Kantone, Krankenkassen, Leistungserbringer) überlassen. Obwohl dabei eine ganze Reihe von Instrumenten entwickelt wurde, mangelt es auch nach über 10 Jahren seit der Einführung des neuen Krankenversicherungsgesetzes in der Schweiz an einem griffigen Konzept, das auf gesamtschweizerisch einheitliche und vergleichbare Art und Weise Erkenntnisse und Aussagen betreffend die Qualität der Spitalbehandlungen ermöglicht.

Aus diesem Grund wurde im Auftrag des zuständigen Departementschefs nach Evaluation von verschiedenen bestehenden Systemen durch das schweizerische Bundesamt für Gesundheit (BAG) ein Pilotprojekt gestartet zur Berechnung und Veröffentlichung von Qualitätsindikatoren im Spitalbereich nach dem Vorbild der Helios Kliniken.

Die Datenlage zur Entwicklung eines solchen Qualitätsmanagements basierend auf Routinedaten ist in der Schweiz sehr gut. Seit dem Jahr 1998 werden im Rahmen der Medizinischen Statistik der Krankenhäuser sämtliche teilstationären und stationären Spitalaufenthalte in der Form eines „patient minimum data set“ erfasst und im Falle der Diagnosen mit ICD-10 respektive der Behandlungen mit der schweizerischen Operationsklassifikation (CHOP) codiert. Ein auf komplexe Art und Weise generierter Hash-Key ermöglicht die anonymisierte, personenbezogene Zusammenführung von einzelnen Episoden zu Patientenpfaden. Auf diese Weise ist auch eine Berechnung von Rehospitalisationsraten möglich.

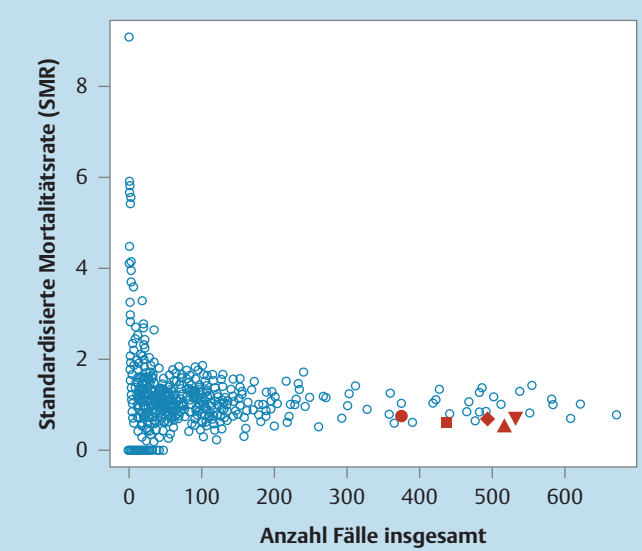

\section{Erste Erfahrungen und Resultate \\ $\nabla$}

Zunächst musste eine auf die in der Schweiz gebräuchlichen Klassifikationssysteme angepasste Indikatorenspezifikation definiert werden. Aufgrund der vorliegenden Zeitreihe der Daten der Medizinischen Statistik von 1998 bis 2006 wurde anschliessend für jedes der 181 Schweizer Akutspitäler ein individualisiertes, 150 Seiten starkes Dossier erstellt, das über die indirekt standardisierten Mortalitätsraten hinaus für jeden Indikator einen Überblick über die medizinische Kodierung gibt der entsprechenden Patientengruppe sowie weitere relevante Merkmale (Abb. 1).

Ziel war es, den Spitälern auf übersichtliche Art und Weise die Möglichkeit zu geben, dem BAG eine Rückmeldung über die Pilotauswertung zu geben. Das Feedback soll Aufschluss geben über die Plausibilität der Auswertungen, eine Stellungnahme seitens der Spitäler betreffend die Datenqualität ihres Betriebs, Aussagen darüber, ob die Auswertungen als nützlich für den internen Verbesserungsprozess angesehen werden und soll den Spitälern eine Möglichkeit geben, Vorschläge zu machen betreffend die Verbesserung des Systems.

Die Evaluation der Behandlungsqualität mittels Mortalitätsraten ist in der Schweiz relativ neu, wie auch die Verwendung der Routinedaten aus der Medizinischen Statistik für den Zweck der Qualitätssicherung. Der Beitrag gibt einen Überblick über die Feedbacks der Spitäler und präsentiert ausgewählte Analysen.

Autorenerklärung: Der Autor erklärt, dass er keine finanziellen Verbindungen mit einer Firma besitzt, deren Produkt in diesem Artikel eine wichtige Rolle spielt oder mit einer Firma, die ein Konkurrenzprodukt vertreibt.

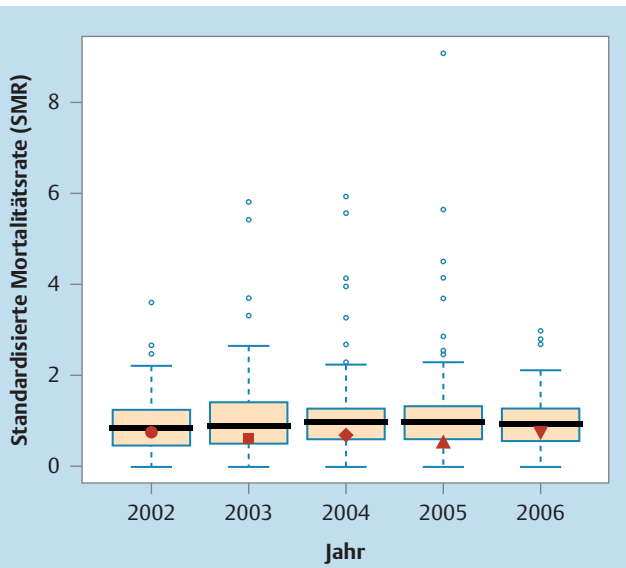

Institut

Schweizerisches Bundesamt für Gesundheit, Bern, Schweiz

Bibliografie

DOI 10.1055/s-0028-1085596

Dtsch Med Wochenschr 2008;

133: S152 . (c) Georg Thieme

Verlag KG Stuttgart - New York ISSN 0012-0472

\section{Korrespondenz}

Dr. phil. Daniel W. Zahnd, MI Schweizerisches Bundesamt für Gesundheit CH-3003 Bern, Schweiz Tel. +41 (0) 313240698 eMail

daniel.zahnd@bag.admin.ch
Abb. 1 Hauptdiagnose Schlaganfall: Überblick über die SMRs von 181 Schweizer Akutspitälern (2002-2006). Beispielhafte Hervorhebung der Längsschnittdaten eines einzelnen Spitals. 